\title{
Os Cuidados Paliativos nos Cuidados de Saúde Primários - o desafio para o século XXI
}

Hélder Aguiar*

\section{RESUMO}

O acentuado envelhecimento populacional que se tem vindo a registar em Portugal coloca desafios ao nível dos cuidados no fim de vida, com reflexo nos Cuidados de Saúde Primários (CSP) e nos médicos de família. Com o advento dos Cuidados Paliativos como área de especialização, foi-se progressivamente adquirindo consciência que o envolvimento dos médicos de família e dos CSP é fundamental para que todos os doentes possam beneficiar deste tipo de cuidados. Existe evidência sólida que este factor é importante para possibilitar a morte no domicílio, o local preferido para a maioria dos europeus, incluindo portugueses. Portugal apresenta uma das mais destacadas taxas de morte em meio hospitalar a nível europeu, reflexo provável, também, do investimento escasso na capacitação dos CSP para o final de vida. A criação do Plano Nacional dos Cuidados Paliativos e da Rede Nacional de Cuidados Continuados (da qual muitos médicos de família já fazem parte), a publicação da Lei de Bases dos Cuidados Paliativos de 5 de Setembro de 2012 e o incentivo às visitas domiciliárias são exemplos de medidas recentes de importância relevante. Porém, existem ainda muitas barreiras por ultrapassar para que cada vez mais médicos de família possam desenvolver cuidados paliativos generalistas com qualidade. Áreas como a formação, a disponibilização de recursos e a articulação com serviços especializados devem ser alvos prioritários. Estando ainda muito longe do padrão de outros países e de se poder oferecer qualidade no final de vida à maioria dos nossos doentes, é um imperativo ético a priorização dos Cuidados Paliativos na agenda da Medicina Geral e Familiar e em cada um de nós.

Palavras-chave: Cuidados Paliativos; Cuidados de Saúde Primários; Medicina Geral e Familiar; Cuidados Paliativos Primários; Portugal.

\section{CONTEXTO: «UM PAÍS QUE (NÃO) É PARA VELHOS»}

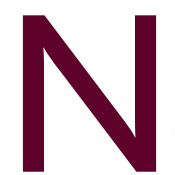
as últimas décadas, o envelhecimento populacional em Portugal atingiu níveis sem precedentes. Na actualidade, é um dos mais destacados a nível mundial. ${ }^{1}$ Segundo os últimos indicadores, o número de idosos já ultrapassa largamente o número de jovens no nosso país (índice de envelhecimento de 120.1 em 2010). ${ }^{2}$ Esta é uma tendência que tende a agravar-se à medida do aumento da esperança média de vida (Portugal ocupa o $14 .^{\circ} \mathrm{e}$ o $23 .^{\circ}$ lugares a nível mundial, em mulheres e homens, respectivamente) e da drástica diminuição da taxa de natalidade. Com apenas 1,3 filhos por mulher em idade fértil, o último relatório das Nações Unidas coloca Por-

*Interno de Medicina Geral e Familiar, USF Vale do Vouga (S. João da Madeira), ACES Aveiro Norte. Mestrando Gulbenkian em Cuidados Paliativos no King's College de Londres. tugal no $2 .^{\circ}$ lugar no topo dos países com menor taxa de fecundidade a nível mundial. ${ }^{1}$

Esta é uma sociedade em transformação. Adaptações nos nossos sistemas organizacionais serão imprescindíveis para evitar o colapso social numa sociedade que se afasta a passos largos do equilíbrio actual. Que necessidades terá esta população? Que tipo de serviços necessitam ser criados?

A população tenderá a morrer em idades mais avançadas. Um estudo recente do Reino Unido prevê que, em 2030, 44\% das mortes ocorram em idosos com 85 anos ou mais. ${ }^{3}$ Com o aumento da idade, doenças crónicas como o cancro, as falências de órgão e as demências (situações de grande fragilidade), terão um peso cada vez maior na nossa sociedade, o que trará enormes desafios ao Sistema Nacional de Saúde (SNS). Será necessária uma adaptação deste a esta realidade, 
contemplando recursos para os cuidados em final de vida.

A prestação de cuidados a estes doentes, em situação de doença avançada ou terminal, tenderá a ser também a realidade dos Cuidados de Saúde Primários (CSP). Estaremos preparados para este desafio?

\section{MEDICINA GERAL E FAMILIAR (MGF) E CUIDADOS PALIATIVOS, DISCIPLINAS TÃO DÍSPARES?}

Apesar dos Cuidados Paliativos, como especialidade, terem emergido em instituições próprias (os hospices), foi progressivamente apercebido que seria remota a possibilidade de todos os doentes em situação terminal terem ou, mesmo, necessitarem do apoio de profissionais especializados. ${ }^{3}$ Efectivamente, a maioria do tempo no ano prévio à morte é passado na comunidade e grande parte dos cuidados aos doentes no último ano de vida tende a ser realizada pelos médicos de família. $^{4}$

Entre as competências definidas para os médicos de família encontra-se a prática longitudinal de cuidados - «um envolvimento que deve ser contínuo desde o nascimento (por vezes antes) até à morte (por vezes depois)». ${ }^{5}$ Este envolvimento, que com naturalidade, surge na prática dos médicos de família, é alicerçado pela existência de vários princípios comuns entre a MGF e os Cuidados Paliativos, entre os quais se incluem: cuidados de saúde centrados na pessoa doente; visão global e holística, integrando aspectos psicossociais e a família; a comunicação como a técnica primordial para atingimento dos objectivos; o meio em que o doente está inserido como aspecto fundamental a capacitar e/ou preservar; acessibilidade e continuidade dos cuidados e o trabalho de equipa interdisciplinar.

Não surpreende que, muito antes da génese dos cuidados paliativos, haja relatos de médicos de família questionando actividades de índole curativa nos seus doentes terminais. No longínquo ano de 1957, Ian Grant, médico de família, descreveu e evidenciou, na sua conduta clínica, os principais princípios que, décadas mais tarde, viriam a definir os cuidados paliativos. ${ }^{6}$ Provavelmente, muitos outros terão tido condutas similares, orientadas por uma visão centrada no doente e que, naturalmente, os aproximou dos padrões de qualidade hoje estabelecidos para os doentes terminais.

\section{AFINAL, QUAL A POSIÇÃO DA MGF NA PRESTAÇÃO DE CUIDADOS PALIATIVOS?}

Apesar da prestação de cuidados a doentes terminais pertencer à identidade dos médicos de família, com o advento dos cuidados paliativos a sua posição na prestação destes cuidados não tem sido consensual, ainda que seja tida como importante. Segundo a Organização Mundial de Saúde (OMS), o envolvimento dos cuidados primários é um processo fundamental para tornar a prática dos cuidados paliativos mais precoce, acessível e global. ${ }^{7}$ No documento que define as normas e padrões para os cuidados paliativos na Europa, a European Association of Palliative Care (EAPC) considera essencial a prática de acções/cuidados paliativos por parte de profissionais com responsabilidades no cuidado a doentes terminais, como os médicos de família. ${ }^{8}$ Esta entidade define de forma clara este papel como Cuidados Paliativos Generalistas ou Acção Paliativa, consoante o envolvimento seja frequente ou ocasional, respectivamente.

A questão fundamental que se coloca actualmente, na era da medicina baseada na evidência, é se a acção dos médicos de família em doentes terminais tem impacto significativo no final de vida destes.

Sabe-se que o envolvimento dos médicos de família, em particular, se associa a uma maior satisfação dos doentes. ${ }^{4}$ Este dado poderá ser justificado parcialmente por outro factor, de extrema importância, que tem merecido destaque na investigação nos últimos anos o local de morte. A capacitação dos doentes para elegerem o local de morte faz parte das directivas avançadas de vida e é cada vez mais um dos aspectos essenciais na qualidade da prestação dos cuidados paliativos.

Estudos observacionais recentes, em vários países desenvolvidos, têm consolidado algo de que já se suspeitava: existe uma associação significativa e independente entre a probabilidade de morte no domicílio e o grau de envolvimento dos médicos de família com doentes terminais. ${ }^{9-13}$ Este envolvimento é caracterizado, sobretudo, pela frequência das visitas domiciliárias. Este dado é tão ou mais importante se atendermos ao facto de a maioria dos europeus, incluindo portugueses, elegerem o domicílio como o local preferido para morrer. ${ }^{14,15}$

Existe, assim, evidência consistente que o envolvimento do médico de família na prestação de cuidados aos doentes terminais da sua lista influencia, de forma positiva e significativa, a vida dos mesmos. 


\section{E EM PORTUGAL, EM QUE SITUAÇÃO ESTAMOS?}

Recentemente, reuniu-se em Vic (Barcelona) uma Taskforce para os Cuidados Primários da EAPC, presidida pelos professores Xaviér Gómez-Baptiste (OMS) e Scott Murray (Universidade de Edimburgo). Neste evento foram apresentados os resultados de um estudo piloto que tinha como objectivo identificar os modelos organizacionais, barreiras e factores facilitadores da prestação de cuidados primários paliativos em vários países da Europa. ${ }^{16,17}$

Um dos resultados deste inquérito piloto revelou que Portugal é um dos países em que se pode identificar maior número de barreiras ao envolvimento dos médicos de família na prestação de acções/cuidados paliativos. Daqui se depreende que, apesar da aproximação cada vez maior às necessidades destes doentes, para o qual contribuíram os incentivos institucionais recentes a visitas domiciliárias, outras medidas terão que ser implementadas.

Por exemplo, a formação em cuidados paliativos é ainda muito escassa em Portugal. Esta barreira dificulta a implementação de equipas e estratégias no território português, negando o acesso a estes cuidados a uma fracção significativa da população. Apesar de recentemente se ter assistido a grandes progressos na acessibilidade dos CSP a medicações mais específicas (como opióides), esta é também e ainda uma dificuldade frequentemente sentida pelos médicos de família. Por outro lado, muitas áreas do território português não são oficialmente cobertas por actividades de apoio e/ou consultadoria por equipas especializadas em cuidados paliativos, o que pode condicionar de forma significativa o envolvimento dos CSP. Nestes, e particularmente na área da prestação de cuidados de saúde a doentes em fim de vida, é comum uma deficiente articulação entre médicos de família, enfermeiros e assistentes sociais e em muitas equipas não existem elementos de grupos profissionais importantes, como a psicologia ou a nutrição. Por último, faltam muitas vezes apoios sociais que permitam à família cuidar dos seus doentes no domicílio.

O local de morte foi outra das variáveis avaliadas por esta Taskforce. Nesta área, Portugal é dos países europeus com uma das maiores percentagens de mortes em meio hospitalar. Verifica-se que esta tendência tem vindo a aumentar - de 2000 a 2008 a percentagem de mor- tes em meio hospitalar aumentou de 54,2 para $61,4 \%$, enquanto as mortes no domicílio (que inclui instituições como lares e residências) diminuíram de 35,8 para $29,9 \%{ }^{18}$ Pode-se perguntar, será sempre sinónimo de qualidade em final de vida a morte em meio hospitalar? Provavelmente não. Especialmente se se tiver em conta que os cuidados paliativos, como área autónoma, ainda não chegaram a muitos hospitais portugueses.

Constata-se, assim, que existe um longo caminho a percorrer no nosso país na área da prestação de cuidados a doentes com doenças crónicas, evolutivas e incuráveis, com várias barreiras por superar. No que diz respeito aos CSP, estes têm um potencial de intervenção considerável, podendo contribuir para reverter a tendência das últimas décadas de morte em meio hospitalar, cumprindo o desejo destes doentes e aumentado a sua qualidade de vida no final.

\section{DE QUE FORMA NÓS, MÉDICOS DE FAMÍLIA, PODEREMOS CONTRIBUIR?}

A evidência que o envolvimento dos médicos de família em acções paliativas resulta em maior satisfação dos doentes e maior probabilidade de morrer em casa deve encorajar o investimento neste recurso, seguindo uma lógica de dignificação da fase terminal de vida e optimização dos recursos de saúde.

A criação do Programa Nacional dos Cuidados Paliativos e o desenvolvimento da Rede Nacional dos Cuidados Continuados têm sido fundamentais porque integram os CSP e a MGF nos seus planos de acção. As Equipas de Cuidados Continuados Integrados (ECCI) têm sido importantes para a criação de equipas domiciliárias dentro dos CSP, ainda que dirigidas a acções de reabilitação em doentes em situação de fragilidade e dependência. Cada vez mais médicos de família fazem parte desta rede e muitos já trabalham nestas equipas diferenciadas. Com a aposta na formação e no aumento da disponibilidade dos médicos de família, poderão ser criadas condições para o aproveitamento do potencial destas equipas domiciliárias também na área dos cuidados paliativos. Outras alternativas possíveis serão a criação de equipas paliativas domiciliárias de base e a extensão ao domicílio das equipas intra-hospitalares. A Lei de Bases dos Cuidados Paliativos, de $15 / 09 / 2012,{ }^{19}$ foi um passo recente importante que permitiu colmatar a ausência dos Cuidados Paliativos na 
legislação portuguesa. Neste documento está definido que uma equipa comunitária de suporte em cuidados paliativos pode estar «integrada numa unidade funcional de cuidados de saúde primários ou na Rede Nacional de Cuidados Continuados Integrados, dispondo de recursos específicos».

De momento, Portugal está muito longe dos padrões encontrados em outros países. Existem apenas 9 equipas domiciliárias reconhecidas pela Associação Portuguesa de Cuidados Paliativos (APCP),,$^{20}$ ou seja, menos de uma equipa por milhão de habitantes, quando a EACP recomenda uma por cada 140 mil habitantes. ${ }^{21} \mathrm{~A}$ crise económica actual deveria, numa perspectiva de optimização de recursos, reforçar a necessidade de formação de mais equipas domiciliárias especializadas em cuidados paliativos. Estudos aleatorizados e controlados identificaram, noutros países desenvolvidos, o contributo destas equipas na redução significativa dos custos com a saúde. ${ }^{22,23}$ Esta redução é consequência, sobretudo, da diminuição do uso de recursos de saúde (idas ao serviço de urgência, dias em internamento) e medicação. No entanto, a maioria do território nacional ainda não dispõe de equipas domiciliárias de cuidados paliativos.

$\mathrm{Na}$ ausência actual destes serviços, muitos médicos de família e equipas de cuidados continuados acabam por providenciar cuidados a doentes terminais. Em todo o caso, é importante preservar e salientar a diferença entre cuidados continuados e cuidados paliativos - reconhecendo nestes casos as limitações (logísticas, recursos, formação) para a prestação dos cuidados adequados aos doentes terminais. Perante esta realidade é especialmente importante que se aposte na articulação entre os CSP, as Unidades de Cuidados Continuados, as ECCI e as equipas intra-hospitalares de cuidados paliativos existentes em Portugal. O protocolo recente entre o Centro Hospitalar de São João e a Administração Regional de Saúde do Norte é um bom exemplo deste tipo de articulação. ${ }^{24}$ Existe evidência que a consultadoria a colegas dos CSP e outros pode, só por si, fazer a diferença. Vários ensaios clínicos aleatorizados, avaliando equipas especializadas em cuidados paliativos puramente de consultadoria (sem intervenção directa nos doentes), identificaram impacto positivo e significativo em áreas como o local de morte ou o controlo de sintomas. ${ }^{25,26,27}$
Por outro lado, o Núcleo de Cuidados Paliativos da Associação Portuguesa de MGF (APMGF) pode ter uma importância crescente no desenvolvimento dos cuidados paliativos na nossa prática clínica. A elaboração das «Recomendações para o tratamento farmacológico da dor $\aleph^{28}$ constitui um bom exemplo, por um lado, de orientações úteis que apoiam a prática do médico de família e, por outro lado, de uma colaboração salutar com a APCP. A elaboração de outros documentos e orientações, em colaboração com a APCP e a DirecçãoGeral de Saúde, poderia ter um impacto relevante na prestação de cuidados aos doentes em fase terminal.

No Reino Unido, no âmbito do programa nacional para os cuidados paliativos, foram criadas ferramentas de apoio à decisão dos médicos de família. Os Gold Standards Framework (GSF) Prognostic Indicator Guidance $^{29}$ são um exemplo emblemático de um algoritmo de aplicação simples e útil para a identificação sistemática dos doentes a necessitar de cuidados paliativos, desenvolvido com o apoio do Royal College of General Practitioners. Outro documento extremamente relevante para os médicos de família em constante actualização - o Liverpool Care Pathway (LCP) ${ }^{30}$ - fornece orientações holísticas para o tratamento do doente terminal nas últimas horas/dias de vida, para médicos e enfermeiros não especializados em cuidados paliativos. Em Portugal existem condições para que as Unidades de Cuidados de Saúde Personalizados e as Unidades de Saúde Familiares possam, através de ferramentas semelhantes adaptadas à nossa realidade, participar ativamente na identificação e organização dos cuidados aos doentes com prognóstico de vida limitado. Actualmente, e perante a desorganização dos cuidados de saúde na fase final de vida, doentes que se encontram nesta situação poderão não ter outra alternativa senão a de recorrer sucessivamente a serviços de urgência, onde são submetidos muitas vezes a tratamentos fúteis e que diminuem a sua qualidade de vida. Frequentemente o passo seguinte é o internamento em serviços hospitalares, a que se segue a morte, em isolamento e sem o controlo efectivo dos sintomas.

Perante esta realidade, parece evidente que a melhoria da prestação de cuidados aos doentes em fim de vida em Portugal depende muito da intervenção da MGF e dos médicos de família. Em média, para listas constituídas por 1700 a 1800 utentes, existirão anual- 
mente cerca de 19 utentes em fase terminal, a maioria destes com necessidades paliativas. Trata-se de um grupo representativo, a necessitar de cuidados especiais. Quer trabalhando com os doentes da nossa lista ou no âmbito de uma equipa domiciliária, não teremos um papel decisivo na qualidade de vida destes doentes?

Está-se perante o desafio de passar das nossas experiências pessoais e isoladas com doentes com patologias avançadas para uma prática mais validada e sistematizada, seguindo o exemplo de outros programas específicos. Será importante o papel dos médicos de família para o estabelecimento e manutenção de padrões de qualidade no seguimento de doentes terminais no ambulatório. Para não se ser «esmagado» pelas exigências de uma sociedade envelhecida e dependente muitas das nossas energias terão que ser direccionadas para este grande desafio. Os cuidados paliativos domiciliários são uma área demasiado importante para ser relegada para segundo plano. Haverá outra área da medicina em que estejamos tão distantes em relação a outros países?

É nesta premissa que se incentiva a que cada vez mais os cuidados paliativos se tornem uma prioridade na agenda dos médicos de família portugueses - seja no local de trabalho, em reuniões de grupo, reuniões com responsáveis hierárquicos, formações pós-graduadas, trabalhos, encontros ou congressos - o contributo de todos poderá fazer, dia após dia, a diferença na vida da população portuguesa que aspira, legitimamente, por melhores cuidados no final de vida.

\section{REFERÊNCIAS BIBLIOGRÁFICAS}

1. United Nations Development Programme (UNDP). Human Development Report, 2011. Disponível em: http://hdr.undp.org/en/media/HDR_ 2011_EN_Complete.pdf [acedido em 05/04/2012].

2. INE. Estatísticas territoriais. Disponível em: http://www.ine.pt/xportal/ xmain? $x p i d=\mid N E \& x p g i d=$ ine_unid_territorial\&menuBOU $\mid=$ 13707095\&contexto=ut\&selTab=tab3 [acedido em 05/04/2012].

3. Franks PJ, Salisbury C, Bosanquet N, Wilkinson EK. Lorentzon M, Kite S, et al. The level of need for palliative care: a systematic review of the literature. Palliat Med 2000 Mar; 14 (2): 93-104.

4. Mitchell GK. How well do general practitioners deliver palliative care? A systematic review. Palliat Med 2002 Nov; 16 (6): 454-64.

5. European Academy of Teachers in General Practice. The European Definition of General Practice / Family Medicine, 2005. Disponível em URL: http://www.woncaeurope.org/Web\%20documents/European\%20Definition\%20of\%20family\%20medicine/Definition\%20EURACTshort\%20version.pdf [acedido em 05/04/2012].
6. Grant I. Care of the Dying. Br Med J 1957 Dec 28; 2 (5060): 1539-40.

7. World Health Organization. Cancer Control - Knowledge into Action. WHO Guide for Effective; 2008. Disponível em: http://www.who.int/ cancer/media/FINAL-Palliative\%20Care\%20Module.pdf [acedido em 05/04/2012].

8. Radbruch L, Payne S. White paper on standards and norms for hospice and palliative care in Europe: part 1. Eur J Palliat Care 2009 Nov-Dec; 16 (6): 278-89.

9. Neergaard MA, Vedsted P, Olesen F, Sokolowski I, Jensen AB, Søndergaard J. Associations between home death and GP involvement in palliative cancer care. Br J Gen Pract 2009 Sep; 59 (566): 671-7.

10. Aabom B, Kragstrup J,Vondeling H, Bakketeig LS, Støvring H. Population-based study of place of death of patients with cancer: implications for GPs. Br J Gen Pract 2005 Sep; 55 (518): 684-9.

11. Fukui S, Fukui N, Kawagoe H. Predictors of place of death for Japanese patients with advanced-stage malignant disease in home care settings: a nationwide survey. Cancer 2004 Jul 15; 101 (2): 421-9.

12. Howat A, Veitch C, Cairns W. A retrospective review of place of death of palliative care patients in regional north Queensland. Palliat Med 2007 Jan; 21 (1): 41-7.

13. Gomes B, Higginson IJ. Factors influencing death at home in terminally ill patients with cancer: systematic review. BMJ 2006 Mar 4; 332 (7540): 515-21.

14. Gomes B, Higginson IJ, Calanzani N, Cohen J, Deliens L, Daveson BA, et al. Preferences for place of death if faced with advanced cancer: a population survey in England, Flanders, Germany, Italy, the Netherlands, Portugal and Spain. Ann Oncol 2012 Aug; 23 (8): 2006-15.

15. Gomes B, Gysels M, Higginson IJ. What is the latest evidence on preferences for place of care and place of death? Palliat Med 2008 Jun; 22 Suppl 1: 465-6.

16. European Association of Palliative Care (EAPC). Palliative Care in Primary Care - in the community. Disponível em URL: http://www.eapcnet.eu/Themes/Organisation/Primarycare.aspx [acedido em 05/04/2012].

17. Murray SA, Sallnow L, Aguiar H. Palliative Care in Primary Care: an update from the taskforce. European Association of Palliative Care Blog, April 13 2012. Disponível em: http://eapcnet.wordpress.com/2012/ 04/13/palliative-care-in-primary-care-an-update-from-the-taskforce/ [acedido em 05/04/2012].

18. Mortality trends in Portugal 2000-2008, INE 2010. Disponível em: http://www.min-saude.pt/NR/rdonlyres/6AB538C0-A7C4-4B5DB4E4-8E46956E4A94/0/estudo_mortalidades.pdf [acedido em 05/04/2012].

19. Diário da República, $1^{\text {a }}$ série $\mathrm{n}^{\circ} 172$. Lei de Bases dos Cuidados Paliativos n 52/2012 de 5 de Setembro. Disponível em: http://www.dre.pt/ pdf1s/2012/09/17200/0511905124.pdf [acediddo em 05/04/2012].

20. Associação Portuguesa de Cuidados Paliativos. Equipas de Cuidados Paliativos. Disponível em: http://www.apcp.com.pt/cuidadospaliativos/ equipasdecuidadospaliativos.html [acedido em 05/04/2012].

21. European Association for Palliative Care (EAPC) Report and Recommendations of a Workshop on Palliative Medicine Education and Training in Europe. Brussels, March 1993. Disponível em: http://www.eapcnet.eu/LinkClick.aspx?fileticket=76YL2TMsDyc\%3D\&tabid=194 [acedido em 05/04/2012]. 
22. Hughes SL, Cummings J, Weaver F, Manheim L, Braun B, Conrad K. A randomized trial of the cost effectiveness of $\mathrm{VA}$ hospital-based home care for the terminally ill. Health Serv Res 1992 Feb; 26 (6): 801-17.

23. Zimmer JG, Groth-Junker A, McCusker J. A randomized controlled study of a home health care team. Am J Public Health 1985 Feb; 75 (2): 134-41.

24. Protocolo de Cooperação Cuidados Paliativos - Região de Saúde do Norte. Disponível em: http://www.apcp.com.pt/uploads/protocoloarschsojoo.pdf [acedido em 05/04/2012].

25. Grande GE, Todd CJ, Barclay SI, Farquhar MC. Does hospital at home for palliative care facilitate death at home? Randomised controlled trial. BMJ 1999 Dec 4; 319 (7223): 1472-5.

26. Rabow MW, Dibble SL, Pantilat SZ, McPhee SJ. The comprehensive care team: a controlled trial of outpatient palliative medicine consultation. Arch Intern Med 2004 Jan 12; 164 (1): 83-91.

27. Jordhøy MS, Fayers P, Saltnes T, Ahlner-Elmqvist M, Jannert M, Kaasa S. A palliative care intervention and death at home: a cluster randomised trial. Lancet 2000 Sep 9; 356 (9233): 888-93.

28. Núcleo de Cuidados Paliativos da Associação Portuguesa de Clínica Geral. Recomendações para o tratamento farmacológico da dor. Rev Port Clin Geral 2007 Jul-Ago; 23 (4): 457-64.
29. The Gold Standards Framework's Prognostic Indicator Guidance (PIG). 4th ed; Oct 2011. Disponível em: http://www.goldstandardsframework.org.uk/Resources/Gold\%20Standards\%20Framework/General/Pr ognostic\%20Indicator\%20Guidance\%20October\%202011.pdf [acedido em 05/04/2012].

30. Liverpool Care Pathway for the Dying Patient. Disponível em: http:// www.mcpcil.org.uk/liverpool-care-pathway/ [acedido em 05/04/2012].

\section{CONFLITO DE INTERESSES}

O autor declara que integra a Unidade de Cuidados na Comunidade Equipa de Cuidados Continuados Integrados de S. João da Madeira, negando outros eventuais conflitos de interesse.

\section{ENDEREÇO PARA CORRESPONDÊNCIA \\ Hélder Aguiar \\ R Condestável 156 \\ 3700-091 S. João da Madeira \\ E-mail: Aguiar.HelderG@gmail.com}

Recebido em 25/04/2012

Aceite para publicação em 13/12/2012

\section{ABSTRACT}

\section{PALLIATIVE CARE IN PRIMARY CARE - THE CHALLENGE FOR THE 21ST CENTURY}

The aging of the Portuguese population poses challenges in end-of-life care for primary care and family physicians. The development of palliative care as an autonomous discipline has led to an understanding that the involvement of primary care and family physicians is essential for the global provision of care. There is evidence that this is an important factor in allowing patients to die at home. This is the preferred place of death for most Europeans, including the Portuguese. Portugal has one of the highest hospital death rates in Europe, possibly reflecting a lack of investment in training primary care physicians for end-oflife care. The creation of the National Plan for Palliative Care, the passage of the Palliative Care Law in Portugal, the creation of the National Network for Continuous Care (in which many family physicians are already taking part), and the provision of incentives for home visits are examples of recent important measures. However, there are still many barriers to overcome to help family physicians improve the quality of palliative care given by generalists. Areas such as training, the availability of resources, and coordination with specialist palliative care should be targeted for improvement. We are still far from the standards set by other European countries in our ability to offer good end-of-life care to our patients. It is ethically imperative to make palliative care a priority on the General Practice agenda in Portugal.

Key-words: Palliative Care; Primary Care; General Practice; General Palliative Care; Portugal. 